\title{
The Regulation of Amino Acid and Sugar Absorption by Diet
}

\author{
Halvor N. Christensen, Ph.D.
}

$\mathbf{F}$ or obvious reasons, nutritionists have traditionally taken a large interest in membrane transport of organic nutrients from the small intestine, i.e., absorption. A second priority has been accorded the parallel subject of the transport of amino acids and sugars into non-epithelial tissues. It is ironic that the interpretation of regulation of nutrient transport has progressed more successfully for the non-intestinal tissue. This paradox can, in retrospect, be accounted for by the earlier discrimination of distinct transport systems in the latter tissues and the consequent earlier identification of systems-specific regulatory processes for them. Here, one may refer to the striking responsiveness of $\mathrm{Na}^{+}$-dependent System A (see Glossary) for neutral amino acids to adaptive regulation, i.e., regulations at the cellular level, and to endocrine regulation. ${ }^{1}$ Even as the application of regulation to other transport systems is currently emerging, the affected systems have usually been concurrently identified.

The observations for nutritional regulation of intestinal amino acid absorption have, in contrast, proceeded with a somewhat hit-or-miss choice of a single amino acid indicator, without recognition of the transport system likely to account for the response. Neame and Wiseman reported in $1959^{2}$ that prior semistarvation, sufficient to decrease the body weight of rats by about one-fifth in 9 days, reversibly accelerated the absorption of both histidine and glucose into everted intestinal sacs by about 80 percent. ${ }^{2,3}$ Scharrer showed, in 1972, that an in-

Halvor N. Christensen, Ph.D., is Professor of Biochemistry in the Department of Biological Chemistry, University of Michigan Medical School, Ann Arbor, MI 48109. crease in dietary protein to 88 percent for two weeks enhanced the subsequent transport of leucine but not of galactose into everted sacs. ${ }^{4}$ Lis, Crampton and Matthews showed, in the same year, ${ }^{5}$ that methionine removal from tied loops of the upper jejunum in the rat was accelerated in vivo not only by restricted food intake, but also by a high-protein or a high-methionine diet. Protein deprivation of 6 to 12 weeks, however, decreased the rate of methionine absorption. The absorption of methionyl-L-methionine, although greater than that of free methionine, was less influenced by dietary changes.

\section{What Transport System Undergoes Regulation?}

To what intestinal transport systems does regulation apply? These results point to two regulatory effects on intestinal amino acid absorption in the rat: 1 ) a stimulation of transport by intervals of protein deprivation and 2) a somewhat specific stimulation of amino acid absorption by high intake of protein or of the test amino acid. Gradually, it was realized that a number of distinct transport systems participate in amino acid transport in the gut. It was also realized that the regulation of absorption could not be understood unless the transport systems concerned are discriminated. In the meantime, the hope has been entertained by some investigators that scattered selections of test amino acids might ultimately point to a clear pattern of modulation of specific transport systems by analogy with systematic transport assignments of the test amino acids possible in various other cells. For example, a stimulation of System L (see Glossary) might conceivably account for responses in all three of the amino acids mentioned in the preceding paragraph. Optimism decreased, however, with the appre- 
Among the transport systems for neutral amino acids, the following may be called public systems, because each serves for numerous amino acids:

System A: Sodium-dependent system notably "uphill," but weaker for amino acids with branched or cyclic side chains. Various regulations converge on this system. Model substrate, 2-(methylamino)isobutyric acid.

System ASC: Sodium-dependent; prefers 3 to 5 carbon atoms in a linear chain and does not tolerate an $\mathrm{N}$-methyl group. Accepts aspartate and its analogues at low $\mathrm{pH}$, hence cysteinesulfinate serves as a model substrate.

System L: Sodium-independent; exchanges amino acids across the membrane; prefers bulkier amino acids, hence bicyclic amino acids serve as models and the bicycloheptane amino acid $(\mathrm{BCH})$ is a probe for this system.

Karasov et al. ${ }^{9}$ use the term private for a transport system used to serve only one or very few amino acids, eg., one serving only for the prolines. These two terms leave in between several systems which one can scarcely call either public or private, hence the public/private classification becomes of little value as we proceed to other systems.

A system specific to glycine and one specific to imino acids, including or (as in a case discussed here) not including glycine, are also encountered. The principal broad-scope sodiumdependent transport activity of the intestinal brush border of the rat seems identical with neither system A nor ASC, although analogy with system $A$ will serve until the system is better characterized. The basolateral pole of the mucosal cell has the usual A/ASC/L systems.

A system for the cationic amino acids collectively (not just arginine or just ornithine) is called system $\mathrm{y}^{+}$, the + sign to indicate the need for a charge on the side chain. More than one system may prove to contribute, as is true in other instances. Citrulline feeding may serve to circumvent a congenital defect in $y^{+}$.

For anionic amino acids, a widely distributed transport system, which accepts either glutamate or aspartate, is conspicuous in the mucosal tissues. The rat hepatocyte also has a system that restricts itself to aspartate and its short analogues, also another system that restricts itself to glutamate and its long analogues. In the $x, y, z$ terminology, these three systems are called $x^{-}{ }_{A G}, X^{-} A, X^{-}$, respectively. If the system is sodium-independent, the $x$ should be lower case. In this terminology, $z$ would refer to zwitterionic, that is, neutral, amino acids.

It will be noted that only occasionally does any single natural amino acid serve to measure accurately the activity of a given transport system. ciation that $\mathrm{Na}^{+}$-dependent transport shows high affinity for a wider range of neutral amino acids in intestinal and renal epithelium than in various other tissues. For example, a $\mathrm{Na}^{+}$. dependent renal resorption was reported even for an artificial bicyclic amino acid elsewhere largely restricted to $\mathrm{Na}^{+}$-independent systems. ${ }^{6}$ Furthermore, the same bicyclic amino acid strongly inhibits a $\mathrm{Na}^{+}$-dependent route of alanine uptake by murine blastocytes not corresponding to System A. ${ }^{7}$ Might this be an early ontologic expression of a principal $\mathrm{Na}^{+}$dependent system of the intestinal brushborder?

Other indications have supported doubts that amino acid absorption could be fitted precisely into the procrustean bed of transport systems tabulated for other cells or tissues. ${ }^{8}$ Part of the difficulty arose from incomplete clarity as to whether transport by the apical or the basolateral pole of epithelial cells, or by both, determined the observed absorption rates. Nev- 
ertheless, the transport systems likely to apply appear to be mainly of the broad-scope types Christensen et al. has described ${ }^{8}$ and which Karasov et al. ${ }^{9}$ have now called public carriers, in contrast with private carriers that serve for only one or two amino acids. In such public systems, essential amino acids presumably share transport with non-essential amino acids and detailed responses appropriate to the economy of each amino acid seem scarcely accessible. Occasionally, evidence has been reported for specificity of regulation ${ }^{10}$ calling for more privacy of transport than has usually been observed.

It has now become apparent that the most immediately useful division of organic nutrient transport can be made between, on the one hand, brush-border transport and, on the other hand, transport across comparatively undifferentiated plasma membrane. This classification places transport across the basolateral surfaces of the epithelial cell provisionally in the same class with transport into a wide range of cells, for example, erythrocytes, fibroblasts and so on. The usual range of amino acid transport systems A, ASC, L and so on (see Glossary) appears to be generally involved in these cells, so that the modes of regulation appear readily accessible to systematic study.

The several methods of study of transport from the intestine or into intestinal tissue have instead usually emphasized overwhelmingly the contribution of the brush border. Only more recently has the technique of preparing vesicles representing selectively the basolateral or brush border portions of the cell surface allowed the demonstration in the basolateral vesicles of the widely distributed types of transport systems seen for other cells, both for monosaccharide and amino acid transport. ${ }^{11,12} \mathrm{~A}$ further method of study now shows its potentiality for offering support: epithelial cell lines from the kidney, when cultured in orientated monolayers, presumably from the proximal tubule, appear to express, for the transport of selected test amino acids, activity only at the basolateral surface. With brush border structures orientated toward the fluid medium and the basolateral surface toward the supporting surface, the monolayer might be expected to act as an epithelium to produce transepithelial transport. Paradoxically, pioneer tests showed evidence for transport of test amino acids in the presumably unphysiological direction, basolateral to brush border. ${ }^{13}$ Furthermore, the $\mathrm{Na}^{+}$. dependent uptake of alanine gave autoradiographic evidence of restriction to the basolateral surface. ${ }^{14}$ Apparently, the opposed transport activity from the brush-border side is not expressed in these cultured cells, predictably leaving unbalanced the net-inward transport from the basolateral surface. This behavior corresponds to the reversible character of intestinal absorption. ${ }^{15}$ The provisional interpretation drawn is that the brush border transport activity may occur by one or more systems not identical with the $\mathrm{Na}^{+}$-dependent systems (presumably including at least $A$ and $A S C$ ) serving for alanine at the basolateral pole.

\section{A New Study}

In this context, a new study of the differential regulation. of proline and glucose transport should be considered. ${ }^{9}$ This study measured the uptake of labeled forms of these two nutrients, each at $50 \mathrm{mM}$, into (not across) an everted $1 \mathrm{~cm}$ sleeve of the mouse intestine secured to a glass rod, with only the mucosal surface exposed. The study chose the mouse rather than the rat, probably an important consideration in view of the differences emerging in the pattern of brush border systems described for the various rodents. ${ }^{16}$ Proline was selected to represent, at least in the rabbit, a comparatively private transport system, the IMINO system, presumably shared only with hydroxyproline among the natural amino acids, but usefully with 2-(methylamino)isobutyric acid as a model amino acid. ${ }^{17}$ (This system should not be confused with the so-called iminoglycine system, described for the rat kidney and other tissues, for which glycine is by definition also a substrate.) Ultimate comparisons of the regulation of "private" with "public" transport systems are an obvious target, along with responses to the economy of essential and the dispensable amino acids.

Uptake was measured for 2 minute intervals for $\mathrm{L}-\left[{ }^{14} \mathrm{C}(\mathrm{U})\right]$ proline, and for 4 minutes for $D-\left[{ }^{14} \mathrm{C}(\mathrm{U})\right]$ glucose. Trace levels of the impermeant probe $\left[1,2-{ }^{3} \mathrm{H}\right]$ polyethylene glycol (M.W. 4,000 ) were used to correct for the substrate 
concentration in adherent fluid. Proline uptake was corrected for the small uptake obtained in the absence of added $\mathrm{Na}^{+}$and D-glucose uptake for the small uptake observed with L-glucose. The $50 \mathrm{mM}$ concentrations yielded uptake rates close to the $V_{\max }$ values. The selected intervals allowed initial rates of uptake to be observed.

The first comparisons were made between mice maintained for at least 14 days either on a chow diet (50 percent carbohydrate; 24.5 percent protein) or a ground beef diet (negligible carbohydrate, protein 65 percent by dry weight), the latter supplemented to 1 percent by the ICN vitamin fortification mixture. This comparison failed to reveal any difference in proline transport, perhaps because the beef diet was only 2.1 times as rich in protein as the chow diet. Therefore, mice in subsequent studies were compared after maintenance for at least 7 days on artificial diets containing 55 percent sucrose plus 15 percent casein, or no sucrose and 70 percent casein. Specific uptake of D-glucose in the proximal jejunum was 68 percent higher in the high-carbohydrate than in the no-carbohydrate mice (also true for the comparison of chow versus beef diets.) For cultured intestinal cells in vitro, a somewhat contrasting result is described below. ${ }^{18}$ The change in proline uptake was just opposite to that for glucose-nearly twice as fast for the no-carbohydrate as for the high-carbohydrate diet. For each solute and diet switch, the stimulation of transport was complete within one day, although the inhibition required several days. Hence, the effects of a given dietary switch to accelerate the uptake of one nutrient and to slow the uptake of the other occurred on different schedules. The results are taken to imply induction and repression of specific intestinal transport carriers by dietary substrate levels. The paper of Karasov et al. ${ }^{9}$ was followed shortly by an independent, largely supportive paper ${ }^{19}$ showing that rat intestinal brush border vesicles derived from animals on a high protein diet took up larger amounts of leucine than controls. This activity was observed when $\mathrm{K}^{+}$at $100 \mathrm{mM}$ both inside and outside the vesicles replaced $\mathrm{Na}^{+}$with or without a gradient. ${ }^{19}$

The authors conclude ${ }^{9}$ that these regulatory effects are also responsible for the gradient ob- served in the transport of the two nutrients along the length of the intestine, that is, decreased rates in both cases as the distance from the pylorus increased. They call attention to related observations of intensified alanine transport into the liver of rats maintained at high protein intakes. ${ }^{20,21}$ In this organ, stimulated alanine uptake was also seen after a 48-hour fast. $^{22}$

Karasov et al. ${ }^{9}$ point out that the half-time for the replacement of intestinal mucosal cells is 17 to 18 hours, whereas one day was required for the stimulation of glucose or proline transport. Accordingly, the changes may depend to uncertain degrees either on the induction of new carrier molecules in existing cells or on the production of new cells richer in these molecules. It should be noted that a regulation of this type would not necessarily be suicidal if it applied to the essential amino acids because there is no evidence that the reduction of proline absorption with decreased protein intake continues to subnormal intakes. Such is not the case, according to prior work, for histidine ${ }^{2,3}$ or methionine. ${ }^{5}$

\section{Difficulties Ahead}

More serious technical problems may be expected in analyzing the regulation of the remarkably wide-range $\mathrm{Na}^{+}$-dependent intestinal transport activity for neutral amino acids, unless a commonality of the regulatory responses identifies one or more homogeneous transport systems. Beyond that possibility, one may have to look to monoclonal antibodies or for common gene determination of the transport of various amino acids to reach an analysis of this component. It may represent two or more heavily overlapping systems. To name the activity now, as though it represented a single transport system, would probably be premature.

Peptide Absorption. Amino acids leave the intestine not only as free amino acids, but also as di- and tri-peptides. ${ }^{23,24}$ These also are transported by a somewhat specific transport system unrelated to the systems serving for free amino acids. An overemphasis of free amino acid absorption in the early decades of this century was no doubt favored by the idea then current that absorption occurs by simple diffu- 
sion, which made it reasonable that digestion products should be brought to their smallest, most diffusible forms. Note that peptides absorbed into the epithelial cell probably exceptionally escape hydrolysis in that cell. Nevertheless, as recently reviewed, 25 the amino acids of peptides infused intravenously are utilized.

The biological regulation of peptide absorption seems not to have been described. This consideration gives special importance to the cited demonstrations of dietary acceleration of the absorption of free proline ${ }^{9}$ and other amino acids; ${ }^{2-5}$ also the failure to find corresponding effects on methionylmethionine absorption. ${ }^{5}$

Sugar Absorption. A precedent for the demonstration of transport facilities for the brushborder surface of intestinal and renal tissues different from those for other cell surfaces had already been seen for the monosaccharides, usually observed with D-glucose. The epithelial cells show a $\mathrm{Na}^{+}$-dependent uphill monosaccharide uptake from the intestinal lumen, whereas the plasma membrane of cells in general (also the basolateral surface of the epithelial cells) shows a $\mathrm{Na}^{+}$-independent apparently passive facilitation of monosaccharide transport. ${ }^{26}$ Furthermore, distinguishable patterns of transporter affinity among the monosaccharides are observed. A single transport system for sugars seems unlikely to account for all observed malabsorption syndromes.

The adaptive and hormonal regulation of monosaccharide transport shares features with that of amino acid transport, but has its own characteristics. Among the most interesting, only recently proposed for amino acid transport, is the recruitment or recycling of transport receptor structures, stored in reserve within the cell interior. ${ }^{27,28}$ The classical example of hormone action on transport, insulin action to stimulate cellular monosaccharide uptake, concerns such recruitment. Moran et al. ${ }^{18}$ report

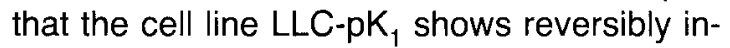
creased capacity for brush-border glucose transport, observed with $\alpha$-methyl-D-glucoside, after culturing a few days in $5 \mathrm{mM}$ rather than $25 \mathrm{mM}$ glucose, ${ }^{18}$ a result somewhat in contrast to the in vivo response seen to high dietary sucrose, compared to no sucrose. ${ }^{9}$

\section{Nutritional Importance of Membrane Transport}

From the first, nutritionists understandably accepted specific membrane transport as part of their subject, given that it serves to bring nutrients from the intestine to the circulation. The similarity of the function of renal tubular resorption and its role in nutrient economy allowed parallel acceptance for that subject. Nutrients have not yet reached the sites where they serve their function, however, when they enter the blood plasma; nor do the various tissues merely "feed at a common trough" of absorbed, circulating nutrients. On the contrary, the movements into various tissues also occur by the same sort of determined, regulatable processes that we see at work in producing intestinal absorption. Nutritionists concede that transport across the placenta ${ }^{29}$ is part of their subject. Why should nutritionists be less willing to accept transport into the hepatocyte or muscle likewise as nutritional subjects? This second stage of transport beyond absorption from the alimentary canal is, by no means, a simple flow of nutrients from the gut to the various tissues. Instead, detailed examination shows interorgan traffic of given nutrients, which can be described as "interorgan nutrition." For example, the intestine receives its nutritive energy as glutamine out of proportion to the glutamine it may receive in the diet. The flow of this glutamine comes largely from the muscles. Other specific flows, for example of alanine and glutamine from the muscle to the liver, have been traced. Other nutrient flows of great interest involve exchanges across the blood-brain barrier. These various flows appear to be regulated to a large degree by actions on the transport processes of the intervening plasma membranes, as this author has had the pleasure of discussing elsewhere. ${ }^{8,30}$ It is interesting to observe that evidence for the uphill transport of nutrients, specifically amino acids, was observed for liver and muscle before it was for the intestine. ${ }^{31}$ (See Classic).

\section{Conclusion}

The small intestine responds both to restricted and to excessive protein intake by increased intestinal amino acid absorption. 
These apparently adaptive regulations demonstrate the range of activities of specific transport systems in the gut. In other tissues, however, knowledge of the regulation of membrane transport has advanced more rapidly. The details of membrane transport and its regulation at the level of molecular biology is gaining recognition as a key subject in nutrition and should presage further scientific advances.

1. G.G. Guidotti, A.F. Borghetti and G.C. Gazzola, Biochim. Biophys. Acta 515: 329-366, 1978

2. K.D. Neame and G. Wiseman, J. Physiol. (London) 146: 10P, 1959

3. T.G. Kershaw, K.D. Neame and G. Wiseman, J. Physiol. (London) 152: 182-190, 1960

4. E. Scharrer, Experientia 28: 267, 1972 .

5. M.T. Lis, R.F. Crampton and D.M. Matthews, Br. J. Nutr. 27: 159-167, 1972

6. K.J. Ulirich, G. Rumrich and S. Klöss, Pflügers Arkiv. Ges. Physiol. 351: 49-60, 1974

7. L.J. Van Winkle, A.L. Campione and M.T. Blood, Fed. Proc. 43: 622, 1984

8. H.N. Christensen, Physiol. Rev. 62: 1193-1233, 1982

9. W.H. Karasov, R.S. Pond III, D.H. Solberg and J.M. Diamond, Proc. Natl. Acad. Sci. USA 80: 7674-7677, 1983

10. R.A. Wapnir, R.L. Hawkins and F. Lifshitz, Am. J. Physiol. 223: 788-793, 1972

11. A.K. Mircheff, C.H. van Os and E.M. Wright, J. Membr. Biol. 52: 83-92, 1980

12. Anonymous, Nutrition Reviews 38: 225-227, 1980

13. C.A. Rabito and M.V. Karish, J. Biol. Chem. 257: 6802-6808, 1982
14. F.V. Sepúlveda and J.D. Pearson, J. Cell. Physiol. 118: 211-217, 1984

15. H.N. Christensen, B.F. Feldman and A.B. Hastings, Am. J. Physiol. 205: 255-260, 1963

16. B.G. Munck in Intestinal Transport. M. GillesBaillien and R. Gilles, Editors, pp. 260-283. Springer-Verlag, Berlin, 1983

17. B.R. Stevens, J.D. Kaunitz and E.M. Wright, Ann. Rev. Physiol. 46: 417-433, 1984

18. A. Moran, R.J. Turner and J.S. Handler, J. Biol. Chem. 258: 15087-15090, 1983

19. S. Wolffram and E. Scharrer, Pflügers Archiv. Eur. J. P Physiol. 400: 34-39, 1984

20. P. Fafournoux, C. Rémésy and C. Demigné, Biochem. J. 210: 645-652, 1983

21. L.Ø. Kristensen, L. Sestoft and M. Folke, Am. J. Physiol. 244: G491-G500, 1983

22. Anonymous, Nutrition Reviews 42: 60-62, 1984

23. Anonymous, Nutrition Reviews 39: 380-383, 1981

24. D.M. Matthews, Biochem. Soc. Trans. 11: 808810,1983

25. L.D. Stegink, Am. J. Dis. Child. 137: 1008-1016, 1983

26. I. Bihler and R. Cybulsky, Biochim. Biophys. Acta 298: 429-436, 1973

27. E. Karnieli, P.J. Hissin, I.A. Simpson, L.B. Salans and S.W. Cushman, J. Clin. Invest. 68: $811-814,1981$

28. T. Kono, Biochem. Soc. Trans. 10: 9-10, 1982

29. Anonymous, Nutrition Reviews 38: 382-384, 1980

30. H.N. Christensen, Bioscience Reports 3: 905913, 1983

31. D.D. Van Slyke and G.M. Meyer, J. Biol. Chem. XVI: 197-212, 1913 